PROCEEDINGS OF THE

AMERICAN MATHEMATICAL SOCIETY

Volume 130, Number 4, Pages 1087-1093

S 0002-9939(01)06195-0

Article electronically published on October 12, 2001

\title{
EXACTNESS OF ONE RELATOR GROUPS
}

\author{
ERIK GUENTNER
}

(Communicated by Joseph A. Ball)

\begin{abstract}
A discrete group $\Gamma$ is $C^{*}$-exact if the reduced crossed product with $\Gamma$ converts a short exact sequence of $\Gamma-C^{*}$-algebras into a short exact sequence of $C^{*}$-algebras. A one relator group is a discrete group $\Gamma$ admitting a presentation $\Gamma=\langle X \mid R\rangle$ where $X$ is a countable set and $R$ is a single word over $X$. In this short paper we prove that all one relator discrete groups are $C^{*}$-exact. Using the Bass-Serre theory we also prove that a countable discrete group $\Gamma$ acting without inversion on a tree is $C^{*}$-exact if the vertex stabilizers of the action are $C^{*}$-exact.
\end{abstract}

\section{INTRODUCTION}

A countable discrete group $\Gamma$ is $C^{*}$-exact if taking the reduced crossed product with $\Gamma$ converts a short exact sequence of $\Gamma$ - $C^{*}$-algebras into a short exact sequence of $C^{*}$-algebras. Classes of discrete groups known to be $C^{*}$-exact include amenable groups [Lan73, word hyperbolic groups Ada94, Ger98], and, by an argument of Connes, discrete subgroups of connected Lie groups [KW99]. The conjecture that all countable discrete groups are $C^{*}$-exact has been disproven by recent work of Gromov, Guentner-Kaminker and Ozawa Gro99, GK99 Oza00 which shows that there exists a countable (even geometrically finite) discrete group that is not $C^{*}$ exact. It is therefore natural to consider the question of which classes of discrete groups are $C^{*}$-exact.

When searching for $C^{*}$-exact groups it is natural to consider groups for which the strong Novikov conjecture has been verified. Indeed, the starting point of the work of Guentner-Kaminker mentioned above was the observation that the classes of groups for which the strong Novikov conjecture has been verified coincides in large measure with the class of groups that are known to be $C^{*}$-exact. Considering the classes of groups mentioned above, the strong Novikov conjecture is known for amenable groups [HK97, HK00], word hyperbolic groups [CM90] and discrete subgroups of connected Lie groups $\mathrm{Kas} 88$. In fact, it is a consequence of the work of Guentner-Kaminker and Ozawa that all $C^{*}$-exact groups satisfy the strong Novikov conjecture.

A one relator group is a discrete group $\Gamma$ admitting a presentation $\langle X \mid R\rangle$, where $X$ is a countable set and $R$ is a single word over $X$. The class of one relator groups is a class for which the Baum-Connes conjecture, and in particular the strong

Received by the editors October 9, 2000.

1991 Mathematics Subject Classification. Primary 47L85; Secondary 20E06, 22D15.

Key words and phrases. Group $C^{*}$-algebra, $C^{*}$-exactness.

The author was supported with funds from the NSF. 
Novikov conjecture, have been verified BBV99, but which are not yet known to be exact. It is the purpose of this brief note to address this gap in the literature. Our first and main result is that countably generated one relator groups are $C^{*}$-exact. We also prove that a countable discrete group $\Gamma$ acting without inversion on a tree is $C^{*}$-exact if and only if the vertex stabilizers of the action are $C^{*}$-exact.

\section{Results}

A countable discrete group $\Gamma$ is $C^{*}$-exact if for every short exact sequence

$$
0 \rightarrow J \rightarrow A \rightarrow B \rightarrow 0
$$

of $\Gamma$ - $C^{*}$-algebras the sequence

$$
0 \rightarrow J \rtimes \Gamma \rightarrow A \rtimes \Gamma \rightarrow B \rtimes \Gamma \rightarrow 0
$$

obtained by taking the reduced crossed product with $\Gamma$ is exact. A $C^{*}$-algebra $D$ is exact if for every short exact sequence of $C^{*}$-algebras as above the sequence

$$
0 \rightarrow J \otimes D \rightarrow A \otimes D \rightarrow B \otimes D \rightarrow 0
$$

obtained by taking the minimal tensor product with $D$ is exact. By a result of Kircheberg-Wassermann, $\Gamma$ is $C^{*}$-exact if and only if its reduced $C^{*}$-algebra $C_{r}^{*}(\Gamma)$ is exact [KW95, Theorem 5.2].

Let $X$ be a countable set of symbols. A word over $X$ is an expression $x_{1}^{n_{1}} \ldots x_{k}^{n_{k}}$ where, for all $1 \leq i \leq k, x_{i} \in X$ and $n_{i}$ is a nonzero integer. The word is reduced if $x_{i} \neq x_{i+1}$, for all $1 \leq i<k$, and cyclically reduced if, in addition, $x_{1} \neq x_{k}$. Its length is $\sum\left|n_{i}\right|$.

A one relator group is a countable discrete group $\Gamma$ that admits a presentation

$$
\Gamma=\langle X \mid R\rangle
$$

where $X$ is a countable set and $R$ is a single word in the elements of $X$. We will call $R$ the relator. Of course, $\Gamma$ is the quotient of the free group generated by $X$ by the smallest normal subgroup containing the relator $R$. We may always replace $R$ by a cyclic permutation without changing $\Gamma$.

Theorem 2.1. Every one relator group is exact.

Induction on the length of the relator $R$ is a fundamental technique for proving results about one relator groups and we will use it to prove the theorem. Following McCool-Schupp [MS73] we give a synopsis of this technique in the following proposition.

Proposition 2.2. Let $\mathcal{C}$ be a class of countable discrete groups containing

(A1) the cyclic groups $\mathbb{Z}$ and $\mathbb{Z} / n \mathbb{Z}, 1 \leq n<\infty$,

and closed under the formation of

(A2) subgroups,

(A3) direct limits,

(A4) amalgamated free products, and

(A5) semi-direct products.

Then $\mathcal{C}$ contains every one relator group. 
A few remarks are in order. By direct limit in (A3) we mean the limit $\Gamma$ of a directed system of the form

$$
\Gamma_{1} \rightarrow \Gamma_{2} \rightarrow \Gamma_{3} \rightarrow \ldots
$$

where all of the maps $\Gamma_{n} \rightarrow \Gamma_{n+1}$ are injective. Thus, $\Gamma=\bigcup \Gamma_{n}$ is the union of a nested sequence of subgroups. From (A1) and (A4) it follows immediately that $\mathcal{C}$ contains the finitely generated free groups $\mathbb{F}_{n}, 1 \leq n<\infty$. From either (A2) or (A3) it then follows that $\mathcal{C}$ contains $\mathbb{F}_{\infty}$, the free group on countably many generators (recall that $\mathbb{F}_{\infty} \subset \mathbb{F}_{2}$ ). We will use these observations below.

Finally, (A4) and (A5) may be replaced by the weaker assumptions

$\left(\mathrm{A} 4^{\prime}\right)$ if $\Gamma_{1}, \Gamma_{2} \in \mathcal{C}$ and $A$ is a free subgroup of each, then $\Gamma_{1} *_{A} \Gamma_{2} \in \mathcal{C}$, and

$\left(\mathrm{A} 5^{\prime}\right)$ if $\mathbb{Z}$ acts on $\Gamma \in \mathcal{C}$, then the semi-direct product $\Gamma \rtimes \mathbb{Z} \in \mathcal{C}$,

respectively. (By a free subgroup we mean a subgroup isomorphic to a finitely or countably generated free group.) These observations will be clear from the proof of the proposition outlined below and may become important when considering stronger approximation properties for one relator groups such as weak amenability (cf. [SS97]).

Assuming the proposition for the moment, the proof of the theorem is immediate.

Proof of Theorem [2.1. It suffices to note that the class of exact groups satisfies conditions (A1)-(A5) of the proposition. Of these, (A1) is immediate.

Properties (A2) and (A3) are also simple. If $\Gamma_{1}$ is a subgroup of $\Gamma$, then $C_{r}^{*}\left(\Gamma_{1}\right) \subset$ $C_{r}^{*}(\Gamma)$. Hence if $\Gamma$ is exact, then so is $\Gamma_{1}$ (see also [KW99, Theorem 4.1]). Also, if $\Gamma$ is the direct limit of the directed system $\Gamma_{1} \rightarrow \Gamma_{2} \rightarrow \ldots$, then $C_{r}^{*}(\Gamma)$ is the direct limit in the sense of $C^{*}$-algebras of the $C_{r}^{*}\left(\Gamma_{n}\right)$. Hence, if each $C_{r}^{*}\left(\Gamma_{n}\right)$ is exact, then so is $C_{r}^{*}(\Gamma)$.

Properties (A4) and (A5) are more difficult, but well-known (see Dyk99, Corollary 3.3] for (A4) and [KW99 Theorem 5.1] for (A5)).

To prove the proposition we require the following simple lemma. We refer to Serre or Baumslag for the definition and basic properties of HNN extensions Ser80. Bau93.

Lemma 2.3. Let $\mathcal{C}^{\prime}$ be a class of countable discrete groups satisfying the conditions (A3)-(A5) of Proposition 2.2. Then $\mathcal{C}^{\prime}$ is closed under the formation of $H N N$ extensions. More precisely, if $A$ is a subgroup of $\Gamma \in \mathcal{C}^{\prime}$ and $\theta: A \rightarrow \Gamma$ is an injective homomorphism, then $H N N(\Gamma, A, \theta) \in \mathcal{C}^{\prime}$.

Proof. As described by Serre [Ser80, p. 8] the given HNN extension is the semidirect product of

$$
\lim \left(\cdots *_{A} \Gamma *_{A} \Gamma *_{A} \Gamma *_{A} \cdots\right)
$$

by $\mathbb{Z}$ for the "shift" action.

We remark that (A5) may be replaced by the weaker (A5') without any change to the lemma or its proof. Further, if (A4) is replaced by the weaker $\left(\mathrm{A} 4^{\prime}\right)$, then the lemma remains true provided we include the assumption that $A$ is a free subgroup of $\Gamma$. 
Proof of Proposition 2.2. The proof is by induction on the length of the relator $R$. If the length of $R$ is zero or one, then $\Gamma$ is a free group and $\Gamma \in \mathcal{C}$.

Suppose that the length of $R$ is strictly greater than one. If $R$ contains only one element from the set $X$, then $R=x^{n}$ for some $x \in X$ and $n \geq 2$. It follows that $\Gamma$ is the free product of $\mathbb{Z} / n \mathbb{Z}$ with a free group and, by (A1), (A4) and the remarks following the statement of the proposition, that $\Gamma \in \mathcal{C}$. We therefore assume that $R$ contains at least two elements from $X$. We introduce the notation $\sigma_{x}(R)$ for the sum of the exponents on the occurrences of the symbol $x \in X$ in $R$ and consider two cases.

Case 1. There exists an $x \in X$ appearing in $R$ such that $\sigma_{x}(R)=0$.

As described by McCool-Schupp [MS73, Theorem 1] $\Gamma$ is an HNN extension $\Gamma=H N N\left(\Gamma^{\prime}, A, \theta\right)$ where

(i) $\Gamma^{\prime}=\left\langle X^{\prime} \mid R^{\prime}\right\rangle$ is a one relator group,

(ii) the length of $R^{\prime}$ over $X^{\prime}$ is strictly smaller than that of $R$ over $X$, and

(iii) $A$ is a countably generated free group.

Thus, by the induction hypothesis $\Gamma^{\prime} \in \mathcal{C}$ and by the lemma $\Gamma \in \mathcal{C}$.

Following McCool-Schupp we describe a presentation of $\Gamma$ from which the above properties may be deduced. Denote the elements of $X$ by $t, b_{0}, c_{0}, \ldots$ where $t$ and $b_{0}$ appear in $R$ and $\sigma_{t}(R)=0$. Replacing $R$ by a cyclic permutation if necessary we assume that the first letter of $R$ is $b_{0}^{ \pm n}$.

Define, for $i \in \mathbb{Z}$,

$$
b_{i}=t^{i} b_{0} t^{-i}, \quad c_{i}=t^{i} c_{0} t^{-i}, \quad \ldots,
$$

and observe that the notation is consistent when $i=0$. The relator $R$ can be rewritten as a word $R^{\prime}$ over the set $\left\{b_{i}, c_{i}, \ldots: i \in \mathbb{Z}\right\}$ of length strictly shorter than the length of $R$ over $X$ according to the following algorithm: replace each symbol $x_{0}^{n}(x=b, c, d, \ldots)$ appearing in $R$ by $x_{i}^{n}$ where $i$ is the sum of the exponents on the occurrences of $t$ appearing to its left (the effect is the removal of all occurrences of $t$ from $R$ ). Noting that $b_{0}$ appears in $R^{\prime}$ define

$$
X^{\prime}=\left\{b_{j}: \alpha \leq j \leq \beta\right\} \cup\left\{c_{i}, d_{i}, \ldots: i \in \mathbb{Z}\right\}
$$

where $\alpha$ and $\beta$ are the minimum and maximum subscripts on $b$ that occur in $R^{\prime}$, respectively. Define $\Gamma^{\prime}$ by the presentation $\Gamma^{\prime}=\left\langle X^{\prime} \mid R^{\prime}\right\rangle$. Define $A$ to be the subgroup of $\Gamma^{\prime}$ generated by the elements of $X^{\prime}$ except $b_{\beta}$, and note that these elements freely generate $A$. Define $\theta: A \rightarrow \Gamma^{\prime}$ by

$$
\begin{aligned}
& \theta\left(b_{j}\right)=b_{j+1}, \quad \alpha \leq j<\beta, \\
& \theta\left(x_{i}\right)=x_{i+1}, \quad i \in \mathbb{Z}, x=c, d, \ldots
\end{aligned}
$$

By considering presentations, we see that $\Gamma \cong H N N\left(\Gamma^{\prime}, A, \theta\right)$.

Case 2. For all $x \in X$ appearing in $R, \sigma_{x}(R) \neq 0$.

In this case, as described by McCool-Schupp [MS73] there exists a discrete group $\Gamma^{\prime \prime}$ such that

(iv) $\Gamma$ is a subgroup of $\Gamma^{\prime \prime}$,

(v) $\Gamma^{\prime \prime}$ is as in Case 1.

Precisely, (v) means that $\Gamma^{\prime \prime}$ is, via the construction of Case 1, an HNN extension $\Gamma^{\prime \prime}=H N N\left(\Gamma^{\prime}, A, \theta\right)$ satisfying (i)-(iii). Hence, $\Gamma^{\prime \prime} \in \mathcal{C}$ and, by (A2), $\Gamma \in \mathcal{C}$. 
Again following McCool-Schupp we describe a presentation for $\Gamma^{\prime \prime}$ from which these statements may be deduced. Denote the elements of $X$ by $a_{0}, b_{0}, c_{0}, \ldots$, where $a_{0}$ and $b_{0}$ occur in $R$. Replacing $R$ by a cyclic permutation if necessary we may assume that $R$ is cyclically reduced with first letter $a_{0}$. Define

$$
X^{\prime \prime}=\left\{t, s, c_{0}, d_{0}, \ldots\right\} .
$$

Let $\alpha=\sigma_{a_{0}}(R)$ and $\beta=\sigma_{b_{0}}(R)$. Let $R^{\prime \prime}$ be the cyclic reduction of the word over $X^{\prime \prime}$ obtained from $R$ by replacing occurrences of $a_{0}$ and $b_{0}$ by $s t^{-\beta}$ and $t^{\alpha}$, respectively. Define

$$
\Gamma^{\prime \prime}=\left\langle X^{\prime \prime} \mid R^{\prime \prime}\right\rangle
$$

It follows easily that $s$ appears in $R^{\prime \prime}$ and that $\sigma_{t}\left(R^{\prime \prime}\right)=-\beta \sigma_{a_{0}}(R)+\alpha \sigma_{b_{0}}(R)=0$. Simple examples show, however, that the length of $R^{\prime \prime}$ with respect to $X^{\prime \prime}$ may indeed be greater than the length of $R$ with respect to $X$. Nevertheless, the construction employed in Case 1 exhibits $\Gamma^{\prime \prime}$ as an HNN extension $\Gamma^{\prime \prime}=H N N\left(\Gamma^{\prime}, A, \theta\right)$, where

$$
\Gamma^{\prime}=\left\langle X^{\prime} \mid R^{\prime}\right\rangle,
$$

$X^{\prime} \subset\left\{s_{i}, c_{i}, d_{i}, \ldots: i \in \mathbb{Z}\right\}$, the length of $R^{\prime}$ with respect to $X^{\prime}$ is strictly less than the length of $R$ with respect to $X$, and $A$ is a free subgroup of $\Gamma^{\prime}$.

We close with a final result concerning $C^{*}$-exactness of countable discrete groups acting on trees. For notation and conventions concerning groups acting on trees, and for fundamentals of the Bass-Serre theory describing the structure of such groups we refer to Serre or Baumslag [Ser80, Bau93].

Theorem 2.4. Let $\Gamma$ be a countable discrete group acting on a tree without inversion. Then $\Gamma$ is $C^{*}$-exact if and only if the vertex stabilizers of the action are $C^{*}$-exact .

Proof. With the following proposition the proof is the same as that of Theorem 2.1

Proposition 2.5. Let $\mathcal{C}$ be a class of countable discrete groups satisfying (A1) to (A5) of Proposition 2.2. Let $\Gamma$ be a countable discrete group acting without inversion on a tree $X$. Then $\Gamma \in \mathcal{C}$ if and only if for all vertices $x \in X$ the stabilizer $\Gamma_{x} \in \mathcal{C}$.

Proof. According to (A2) if $\Gamma \in \mathcal{C}$, then $\Gamma_{x} \in \mathcal{C}$ for all vertices $x \in X$. The proof of the converse proceeds (compare CCJ+98 Sec. 5.2, Theorem 10]) using the BasseSerre theory for groups acting without inversion on trees according to which $\Gamma$ is the fundamental group of a graph of groups $G$ over $Y=X / \Gamma$ satisfying

$G_{v} \cong$ the stabilizer in $\Gamma$ of $\widetilde{v}$, a vertex of $X$ maping to the vertex $v$ of $Y$,

$G_{e} \cong$ the stabilizer in $\Gamma$ of $\widetilde{e}$, an edge of $X$ mapping to the edge $e$ of $Y$.

By assumption the groups $G_{v}$, and hence also their subgroups the $G_{e}$, are in $\mathcal{C}$. Note further that this is the only property we use subsequently; we are not concerned with the precise choices needed to ensure that the fundamental group of $G$ is $\Gamma$. In fact, we prove that if $G$ is a graph of groups from $\mathcal{C}$, then the fundamental group $\Gamma$ of $G$ is also in $\mathcal{C}$. This we do by considering two cases.

Case 1. $Y$ is finite. In this case $\Gamma$ is constructed from the $G_{v}$ and $G_{e}$ by a finite sequence of amalgams and HNN extensions (see [Bau93, VII.5]). Hence, if the $G_{v}$ and $G_{e}$ are in $\mathcal{C}$, so is $\Gamma$ by (A4) and Lemma 2.3 
Case 2. $Y$ is general. Observe that $\Gamma$ is the direct limit of the fundamental groups of the finite subgraphs of $Y$ (this is best seen using the "normal form" for elements of $\Gamma$ Bau93 VII.6]). By (A3) and the previous case, $\Gamma$ is in $\mathcal{C}$.

We obtain the following corollaries [GK99, GK00, Oza00]:

Corollary 2.6. Let $\Gamma$ be a finitely generated one relator group. Then $\Gamma$ is uniformly embeddable in a Hilbert space and further acts amenably on a compact space.

Corollary 2.7. Let $\Gamma$ act on a tree without inversion. If every vertex stabilizer acts amenably on a compact space, then so does $\Gamma$.

Added note. After this paper was submitted I learned that in his work on Property A Tu has independently obtained results similar to Theorem 2.4 [Tu00].

\section{REFERENCES}

[Ada94] S. Adams, Boundary amenability for hyperbolic groups and an application to smooth dynamics of simple groups, Topology 33 (1994), 765-783. MR 96g:58104

[ADR98] C. Anantharaman-Delaroche and J. Renault, Amenable groupoids, Monographies de L'Enseignement Math. 36, Geneva, 2000. CMP 2001:05

[Bau93] G. Baumslag, Topics in combinatorial group theory, ETH Lectures in Mathematics, Birkhäuser, Boston, 1993. MR 94j:20034

[BBV99] C. Beguin, H. Bettaieb, and A. Valette, $K$-theory for $C^{*}$-algebras of one-relator groups, $K$-Theory 16 (1999), 277-298. MR 2000c: 46133

[CCJ+98] P. Cherix, M. Cowling, P. Jollissaint, P. Julg, and A. Valette, Locally compact groups with the Haagerup property, Unpublished manuscript, 1998.

[CM90] A. Connes and H. Moscovici, Cyclic cohomology, the Novikov conjecture, and hyperbolic groups, Topology 29 (1990), 345-388. MR 92a:58137

[Dyk99] K. Dykema, Exactness of reduced amalgamated free product $C^{*}$-algebras, Preprint, 1999.

[Ger98] E. Germain, Approximate invariant means for boundary actions of hyperbolic groups, Appendix to Amenable Groupoids [ADR98], 1998. CMP 2001:05

[GK99] E. Guentner and J. Kaminker, Exactness and the Novikov conjecture, To appear in Topology, 1999.

[GK00] E. Guentner and J. Kaminker, Addendum to "Exactness and the Novikov conjecture", To appear in Topology, 2000.

[Gro99] M. Gromov, Spaces and questions, Unpublished manuscript, 1999.

[HK97] N. Higson and G. G. Kasparov, Operator K-theory for groups which act properly and isometrically on Hilbert space, Electron. Res. Announc. Amer. Math. Soc. 3 (1997), 131-142. MR 99e:46090

[HK00] N. Higson and G. G. Kasparov, E-theory and KK-theory for groups which act properly and isometrically on Hilbert space, To appear in Invent. Math., 2000.

[Kas88] G. G. Kasparov, Equivariant $K K$-theory and the Novikov conjecture, Invent. Math. 91 (1988), 147-201. MR 88j:58123

[KW95] E. Kirchberg and S. Wassermann, Operations on continuous bundles of $C^{*}$-algebras, Mathematische Annalen 303 (1995), 677-697. MR 96j:46057

[KW99] E. Kirchberg and S. Wassermann, Permanence properties of $C^{*}$-exact groups, Documenta Mathematica 4 (1999), 513-558. CMP 2000:05

[Lan73] L. Lance, On nuclear $C^{*}$-algebras, J. Funct. Anal. 12 (1973), 157-176.

[MS73] J. McCool and P. Schupp, On one relator groups and HNN extensions, J. of the Australian Math. Society 16 (1973), 249-256. MR 49:2952

[Oza00] N. Ozawa, Amenable actions and exactness for discrete groups, C. R. Acad. Sci. Paris Ser. I Math. 330 (2000), No. 8, 691-695. CMP 2000:14

[Ser80] J. P. Serre, Trees, Springer, New York, 1980, Translation from French of "Arbres, Amalgames, $S L_{2}$ ", Astérisque no. 46. MR 82c:20083 MR 57:16426 
[SS97] A. M. Sinclair and R. R. Smith, The completely bounded approximation property for discrete crossed products, Indiana Univ. Math. J. 46 (1997), 1311-1322. MR 99e:46072

[Tu00] J. L. Tu, Remarks On Yu's Property A for discrete metric spaces and groups, Preprint, 2000 .

Department of Mathematical Sciences, Indiana University-Purdue University Indianapolis, 402 N. Blackford St., Indianapolis, Indiana 46202-3216

Current address: Mathematical Sciences Research Institute, 100 Centennial Drive, \#5070, Berkeley, California 94702-5070

E-mail address: guentner@msri.org 